\title{
A Serious Diagnosis often Missed: Salicylate Induced Pulmonary Edema (SIPE)
}

\author{
Lakshmi Kant Pathak $^{1, *}$, Vimala Vijayaraghavan ${ }^{2}$, Chirag Chavda ${ }^{3}$ \\ ${ }^{1}$ Department of Medicine, Sanford Medical Center University of North Dakota, USA \\ ${ }^{2}$ Caribbean Medical University, Chicago Campus, USA \\ ${ }^{3}$ Paris Regional Medical Center, Paris, Texas, USA \\ *Corresponding author: drpathaklk@gmail.com
}

Received January 10, 2015; Revised January 17, 2015; Accepted January 21, 2015

\begin{abstract}
Salicylate induced pulmonary edema is mostly a un recognized diagnosis as most physicians have little or no experience in treating it. Therefore there are only 140 possible case reports of SIPE in literature making it a rare entity. Failure to recognize this condition may lead to significant morbidity and mortality. Therefore the objective of this case report is to make aware of the possibility of this diagnosis in patients presenting with neurological changes, fever, and dyspnea and anion-gap metabolic acidosis. It also discusses the best current practice guidelines on treatment of this condition based on present literature. A more definitive guideline on its diagnosis and treatment needs further research and study.
\end{abstract}

Keywords: SIPE, pulmonary edema

Cite This Article: Lakshmi Kant Pathak, Vimala Vijayaraghavan, and Chirag Chavda, “A Serious Diagnosis often Missed: Salicylate Induced Pulmonary Edema (SIPE).” American Journal of Medical Case Reports, vol. 3, no. 2 (2015): 37-39. doi: 10.12691/ajmcr-3-2-5.

\section{Introduction}

Salicylate induced pulmonary edema (SIPE) is a complication of salicylate toxicity in both acute and chronic salicylate or aspirin users. The diagnosis is most often missed due to inability to recognize the clues compounded with the fact that most patients have associate neurological symptoms which make difficult to obtain a reliable history. Patients are often mis-diagnosed and treated for other conditions including pneumonia, heart failure and sepsis. This leads to significant delay and may cause severe morbidity and mortality.

\section{Case Report}

A 37 year old male patient presented to our emergency department with symptoms of altered mental status, tachypnea, and moderate respiratory distress. He was febrile with temperature of 101 degree Fahrenheit and hypoxic on room air. He reported taking "a bunch of aspirins” for his chronic back pain. On further questioning, he stated that for the past 3 months, he had been taking, on average, five to six of the over the counter Bayer Back and Body tablets each containing $500 \mathrm{mg}$ of aspirin. Three to four days prior to presentation, he started taking about eight to ten tablets daily as his back pain worsened.

His medical history included hypertension and he smokes one pack of cigarettes every day.

Pertinent Physical exam findings included tachypnea with a respiratory rate of $45-50$ breaths/minute with oxygen saturation of $90 \%$ after he had been on non-invasive ventilator for 30 minutes with a FiO2 of $100 \%$. He was confused to time and place but without any focal motor weakness.

Lungs revealed extensive bilateral crepitation. Heart sounds was normal and the abdominal exam benign.

Pertinent abnormalities on laboratory evaluation included bicarbonate of $11 \mathrm{mmol} / \mathrm{L}$, creatinine of 2.37 $\mathrm{mg} / \mathrm{dl}$ and a salicylate level of $51 \mathrm{mg} / \mathrm{dl}$. ABG on $100 \%$ FiO2 was $\mathrm{pH}$ of $7.37, \mathrm{PaCO} 2$ of $18 \mathrm{~mm} \mathrm{Hg}, \mathrm{PaO} 2$ of $73 \mathrm{~mm} \mathrm{Hg.}$

Chest X-ray and CT scan of the chest are as showed below:

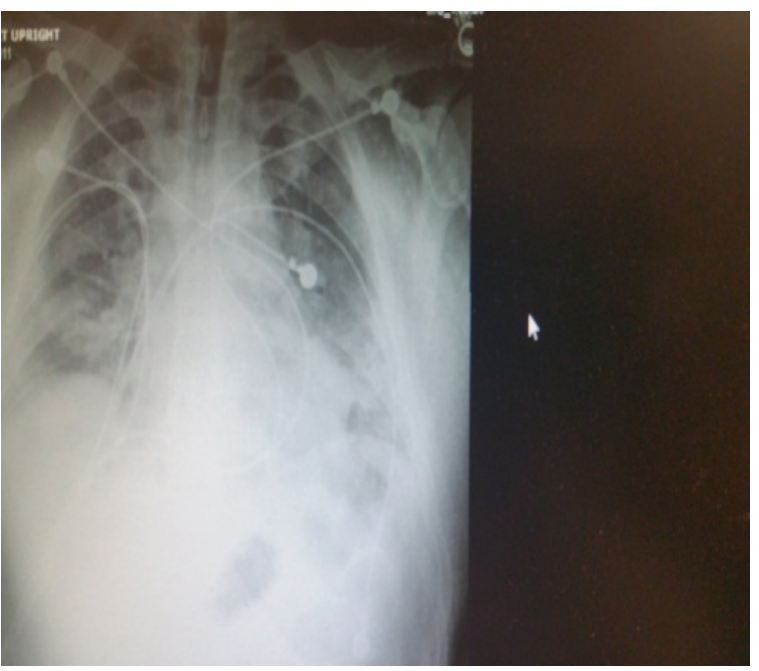

Picture A. Chest X Ray showing pulmonary edema 


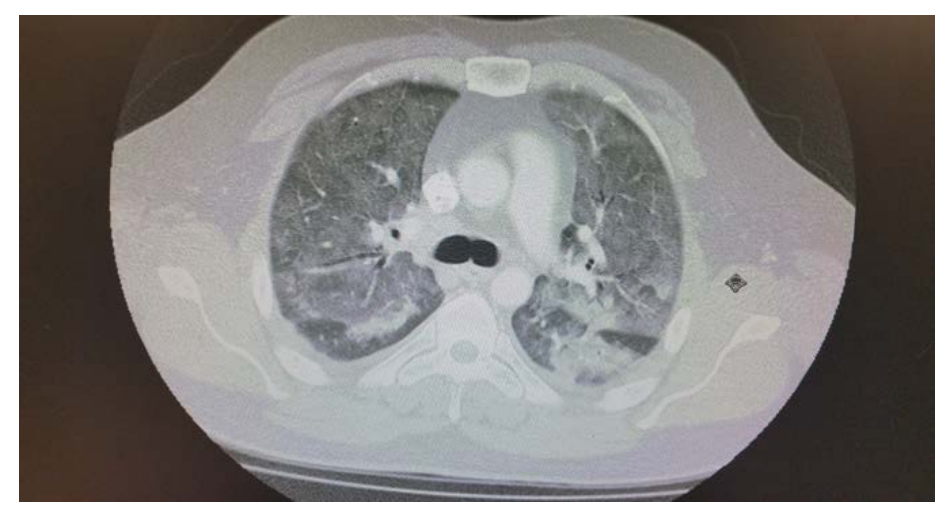

Picture B. CT chest showing extensive bilateral ground glass appearance

Echocardiogram showed preserved left and right ventricular function and was completely normal.

Based on clinical findings a diagnosis of SIPE was made and nephrology was consulted for hemodialysis.

Although the current guidelines recommend urinary alkalization with intravenous sodium bicarbonate as best treatment strategy but it is not clear if such treatment will further worsen pulmonary edema and respiratory failure.

The patient underwent emergency hemodialysis for one session. He was continued on non-invasive ventilation and eventually had a complete recovery clinically and biochemically.

\section{Discussion}

Salicylate induced non cardiogenic pulmonary edema must be considered in patients with history of acute or chronic salicylate ingestion. It symptoms and findings are often misleading to other possible diagnoses like pneumonia, heart failure, ARDS, pulmonary embolism and sepsis syndrome.

A careful history is important to elicit although due to mental state changes it is always not possible.

Any patient who presents with tachypnea, fever, neuropsychiatric changes, anion gap metabolic acidosis and respiratory alkalosis with history of salicylate ingestion, this should be kept as high possibility.

The key to the diagnosis is to consider all possible causes of anion gap metabolic acidosis and eliminate each one of them, in absence of a good history like for example, DKA, uremia, lactic acidosis, sepsis and aspirin toxicity.

Though there are many case reports published in literature there is still a debate on the best practice guidelines on its early diagnosis and treatment.

An extensive review article published in Medscape has found multiple confounding factors among the 139 cases of possible SIPE. Many had multiple drug ingestion including codeine and benzodiazepines. There was no clarity on the definition of acute versus chronic ingestion of salicylates [1].

Many of the patients developed pulmonary edema after forced alkaline diuresis and many had cardiac cause for pulmonary edema. Few patients had SIRS [2] and in few cases it was ARDS caused by sepsis syndrome [3].

There is suggestion that age more than 30years, smoking, chronic salicylate ingestion, metabolic acidosis and CNS symptoms on admission are strong risk factors for the development of SIPE, however the data is inconclusive [4,5].
Similarly the studies also fail to discuss optimum treatment strategy and clear protocol.

A recent guideline by the American Association of Poison Control Centers suggest acute toxicity as ingestion of less than eight hours and chronic as longer than this duration [6].

The cause for pulmonary edema in SIPE is an increase in capillary permeability of the lungs leading to protein leakage and transudation of fluid in both pulmonary and renal tissues. Change in Prostacyclin's caused by salicylates also plays a role [7].

\section{Diagnostic Evaluation}

Initial investigation should include $\mathrm{CBC}, \mathrm{BMP}, \mathrm{ABG}$ urine analysis, drug screen, chest x-ray, ECHO and may be CT chest. In addition where salicylate toxicity is uncertain as cause if SIPE, bronchoscopy may help to rule out other possible etiologies like acute interstitial pneumonia, pulmonary hemorrhage hypersensitivity pneumonitis and and eosiniphilic pneumonia. This is also important as the treatment for this condition is different from SIPE and delay in diagnosis may affect the outcome.

\section{Treatment}

The best treatment guideline are yet to be made but urinary alkalization with intravenous bicarbonate is considered first line therapy as it enhances urinary elimination of salicylate although this increase the risk of worsening pulmonary edema. Correction of dehydration and electrolyte replacement are important too. The target $\mathrm{pH}$ for serum alkalization is 7.5-7.55. In intubated patients hyperventilation is necessary to maintain hyppocarbia and alkalemia.

The treatment is guided by serum $\mathrm{pH}$ and salicylate concentration every two hours along with clinical improvement. A mild change in renal clearance in chronic users can cause big decline in clinical condition as salicylate binding sites are already saturated.

No clear guidelines exists when to use Hemodialysis in SIPE. The data so far collected from studies are insufficient. However its use is suggested in patients with pulmonary edema, neuropsychiatric changes and impaired renal function (). There is also reference to use it when the salicylates levels are very high $>100 \mathrm{mg} / \mathrm{dl}$ regardless of the clinical presentation and at lower levels in chronic users due to saturation of protein binding sites. 
It is a valuable option early on in the treatment to prevent organ damage and mortality.

\section{References}

[1] James K.glisson, MD, Telciane S.Vesa, MD. Current Management of Salicylate-induced pulmonary edema. South Med J.2011; 104(3): 225-232.

[2] Chalasani N, Roman J, Jurado RL. Systemic inflammatory response syndrome caused by chronic salicylate intoxication. South Med J 1996; 89: 479-482.

[3] Thisted B, Krantz T, Strøm J, et al. Acute salicylate self-poisoning in 177 consecutive patients treated in ICU. Acta Anaesthesiol Scand 1987; 31: 312-316.
[4] Zimmerman GA, Clemmer TP. Acute respiratory failure during therapy for salicylate intoxication. Ann Emerg Med1981; 10: 104106.

[5] Niehoff JM, Baltatzis PA. Adult respiratory distress syndrome induced by salicylate toxicity. Postgrad Med1985; 78: 117-119, 123.

[6] Chyka PA, Erdman AR, Christianson G, et al. Salicylate poisoning: an evidence-based consensus guideline for out-of-the-hospital management. Clin Toxicol (Phila) 2007; 45: 95-131.

[7] Kerr F, Krenzelok EP. Salicylates, in Shannon M, Borron SW, Burns M (eds): Haddad and Winchester's Clinical Management of Poisoning and Drug Overdose. Philadelphia, Sanders 2007, ed 4, pp 835-848. 